\title{
Parametric study of the behaviour of group velocity dispersion in optical pulse compression
}

\author{
R.F.X.A.M. Mols and G.J. Ernst \\ University of Twente, Department of Applied Physics, P.O. Box 217, 7500 AE Enschede, The Netherlands
}

Received 16 March 1992; revised manuscript received 17 August 1992

\begin{abstract}
An optical pulse compressor is investigated over a large range of compression factors, fiber lengths and laser powers. The given theory for zero group velocity dispersion is valid over the investigated range of grating-prism distances. The influence of stimulated Raman scattering limited the feasible compression factors due to instabilities of the output pulse. For long fiber lengths it is experimentally shown that group velocity dispersion is present while the theory of zero group velocity dispersion still predicts the right pulse duration. The advantage of the presence of group velocity dispersion is the improved beam quality. The optical pulse compressor investigated uses a mode-locked Nd:YLF laser followed by an adjustable fiber-grating compressor stage.
\end{abstract}

\section{Introduction}

A laser system giving stable pulses with tunable length is needed for a photocathode driven linac. This linac is used as an injector for the TEU-FEL project [1]. The advantages of such an injector are its high current, low emittance and low energy spread. The electrons are generated by irradiation of a photocathode in an RF cavity [2]. The properties of this electron beam are therefore controlled by the temporal and spatial shape of the light pulse. The pulse length might be controlled by compression of the 50 ps pulses of a mode-locked Nd:YLF laser. Until now, compressors consisting of a fiber-grating setup are used mainly to compress pulses as far as possible to get a maximum peak power of the shortest pulse length. The former often use Nd:YAG lasers, in combination with chirped pulse amplification in a regenerative glass-amplifier setup [3], while the latter use CPM lasers of which the pulses are compressed to a few femtoseconds [4]. In this work we present a parametric study of the compressor to investigate the behaviour of the output pulses.

* Sponsored by the Nederlands Centrum voor Laser Research (NCLR), the Dutch Foundation for Technological Research (STW) and the Dutch Foundation for Research on Matter (FOM).

\section{Theory}

The light of a laser source is first coupled into a fiber in which the width of the frequency spectrum is increased due to the effect of self phase modulation (SPM). The group velocity dispersion (GVD) of the fiber tends to linearise the frequency chirp of the pulse. This pulse is compressed after the fiber using a negative dispersive optical path.

First, the individual action on a pulse due to GVD and SPM is investigated using characteristic propagation lengths. These lengths are defined as the length in a medium with SPM or GVD needed to increase the bandwidth-time product by $\sqrt{2}$. The meaning of the various constants or parameters used are given in table 1.

Due to GVD the pulse is spread in time caused by the different group velocities for the various frequency components in the pulse. The characteristic length needed to increase the pulse width by a factor $\sqrt{2}$ is [5]

$Z_{\mathrm{D}}=\frac{8 \ln 2}{n \lambda D}\left(\frac{\pi c}{\Delta \omega}\right)^{2}$

which decreases when the GVD parameter

$D \equiv \frac{\omega_{0}}{v_{\mathrm{p}}} \frac{\mathrm{d}}{\mathrm{d} \omega}\left(\frac{1}{v_{\mathrm{g}}}\right)$, 
Table 1

Various constants concerning the Nd:YLF laser, the fiber and the grating.

\begin{tabular}{lll}
\hline Initial pulse length & $\tau_{0}$ & $50 \mathrm{ps}$ \\
Refractive index fiber & $n$ & 1.6 \\
Nonlinear coefficient & $n_{2}$ & $6.2 \times 10^{-20} \mathrm{~m}^{2} / \mathrm{W}$ \\
GVD parameter & $D$ & $2.4 \times 10^{-3}$ \\
Effective core diameter & $d$ & $7.7 \mu \mathrm{m}$ \\
Raman gain coefficient & $g$ & $1 \times 10^{-13} \mathrm{~m} / \mathrm{W}$ \\
Wavelength in vacuum & $\lambda$ & $1053 \mathrm{~nm}$ \\
Groove spacing of grating & $a$ & $1 / 1800 \mathrm{~mm}$ \\
Velocity of light & $c$ & $3 \times 10^{8} \mathrm{~m} / \mathrm{s}$ \\
Fiber parameter & $\zeta$ & $425 \mathrm{~m} \mathrm{~W}$ \\
\hline
\end{tabular}

with $v_{\mathrm{p}}$ the phase and $v_{\mathrm{g}}$ the group velocity, of the fiber or the spectral width $\Delta \omega$ of the pulse increases. The effect of SPM causes the generation of new frequency components $\omega_{\mathrm{i}}(t)=\mathrm{d} \phi(t) / \mathrm{d} t$ where the phase of the pulse is given by $\phi(t)=2 \pi\left[n+n_{2} I(t)\right] L /$ $\lambda$, in which $n_{2}$ is the intensity $(I(t))$ dependent part of the overall index of refraction $n+n_{2} I(t)$ and $L$ is the length of the medium. For a gaussian pulse shape $I(t)=I_{0} \exp \left[-4 \ln 2\left(t / \tau_{0}\right)^{2}\right]$ which is assumed not to be altered by SPM, the evolution of the spectral width $\Delta \omega$ is calculated, defining $\Delta \omega=2 \omega_{\mathrm{i}}\left(\tau_{0}\right)$, as

$\Delta \omega=2 \pi \ln 2 \frac{n_{2} I_{0} L}{\lambda \tau_{0}}$.

This expression may also be used to calculate the characteristic length $\left(Z_{\mathrm{s}}\right)$ due to SPM:

$Z_{\mathrm{s}}=\frac{\sqrt{2}}{2 \pi \ln 2} \frac{\lambda}{n_{2} I_{0}}$.

This characteristic length can be interpreted as a threshold condition for SPM. The existence of SPM depends on the length of the fiber $z$ and the power of the pulse $P=\pi I_{0}(d / 2)^{2}$. SPM will therefore occur when

$P z>\frac{\sqrt{2}}{8 \ln 2} \frac{\lambda d^{2}}{n_{2}}$.

For a typical experimental value of $I_{0}=0.5 \mathrm{GW} / \mathrm{cm}^{2}$ this means that SPM occurs when $z>0.2 \mathrm{~m}$. Due to the spectral broadening the maximum pulse compression $\left(\tau / \tau_{0}\right)$, where $\tau$ is the compressed pulse length, can be estimated by looking at the creation of new frequencies $\Delta \omega$ :

$\tau_{0} / \tau=1+\tau_{0} \Delta \omega=1+2.3 P L / \zeta$.
Combined SPM and GVD is expected when their characteristic lengths are of the same order of magnitude. From eq. (3) it is seen that the characteristic length for SPM only depends on $\lambda$ and $I_{0}$. In our experimental setup a typical value for $I_{0}$ is $0.5 \mathrm{GW} /$ $\mathrm{cm}^{2}$ which gives an order of magnitude for $Z_{\mathrm{s}}=0.2$ $\mathrm{m}$. At the beginning of the fiber, $\Delta \omega=55 \mathrm{GHz}$ giving a characteristic length for GVD as $Z_{D} \sim 400 \mathrm{~km}$. However, eq. (1) shows that the characteristic length for GVD is dependent on $\Delta \omega$ and thus is a dynamical variable. Since the spectral width increases due to SPM the lengths $L$ of a fiber needed to increase the spectral width in order to get $Z_{\mathrm{s}}=Z_{\mathrm{D}}$ is calculated to be

$L^{2}=\frac{8 \pi}{\sqrt{2}} \frac{c^{2} \tau_{0}^{2}}{n D n_{2} I_{0}}$.

Using the parameters of table 1 and again $I_{0}=0.5$ $\mathrm{GW} / \mathrm{cm}^{2}$ this length is $L \sim 1800 \mathrm{~m}$. In our measurements the fiber lengths will be shorter than $125 \mathrm{~m}$ and GVD is negligible.

The analysis given above only gives some orders of magnitude but is a strong tool to compare the characteristic lengths of the individual processes GVD and SPM. For a more detailed comparison between experiment and theory we need to solve the nonlinear Schrödinger equation [5] which describes the propagation of a pulse in a medium with combined SPM and GVD. A numerical study of this equation [6] assuming a sech ${ }^{2}$ initial pulse shape showed that the influence of GVD is negligible compared to the action of SPM, which means $Z_{\mathrm{D}}<Z_{\mathrm{s}}$, when

$P z / \zeta>3$

where properties of the fiber are characterized by $\zeta$,

$\zeta=8.8 \times 10^{-10} \frac{n c \lambda d^{2}}{n_{2}}(\mathrm{~W} \mathrm{~m})$.

This condition is the same as the condition for SPM given above in condition (4) except for the numerical constant. Under these circumstances, i.e. zero GVD and SPM above threshold, the optimum compression of a setup consisting of the fiber and a negative dispersive path is estimated [6]:

$\tau_{0} / \tau=1+0.9 P z / \zeta$ 
The difference between our approximation (5) and this numerical result is due to the frequency chirp of the pulses. The chirp of the pulses is nonlinear and therefore this chirp is not completely compensated for in the negative dispersive path which in the case of a grating or prism pair only compensates the linear part of the frequency chirp. From the relations given above we see that the compression inefficiency is about 2.5 times. This behaviour is the same as observed by Tomlinson [6], they calculated a factor of four for the inefficiency of the total compression factor. Due to the nonlinear chirp they observed side lobs in the time domain.

To achieve the optimum compression with a dispersive path which consists of two parallel gatings, the distance $(b)$ between the gratings is estimated by [6]

$b=\frac{\tau_{0} \zeta}{1.76} \frac{4 \pi c^{2} a^{2}}{P z \lambda^{3}}$.

Condition (7) contains the same term as eq. (9), therefore we rewrite this condition in terms of the compression factor. The condition is then valid for compression factors more than about four, independent of the used fiber, the fiber length or the laser power. The grating-grating distance at maximum compression is in this case about $25 \mathrm{~cm}$.

An optimized compression is expected when the fiber length is longer than $1500 \mathrm{~m}$ because GVD tends to linearise the frequency chirp. However, from ref. [6] it is found that this optimum occurs for a fiber length of $\sim 800 \mathrm{~m}$. So this it is clear that even a small influence of GVD has a large influence on the chirp. The resulting pulse length after compression will be $\sim 0.5$ ps. However, such a compression can never be reached due to stimulated Raman scattering (SRS), which starts at the critical power $P_{\mathrm{c}}$, estimated by [7]

$g P_{\mathrm{c}} z \approx 16 \pi(d / 2)^{2}$,

where the factor 16 gets closer to 20 for fibers of only a few meters. When SRS occurs the pulse becomes unstable since SRS starts up from noise. Since this is unwanted in most applications stable compression is expected when for a given fiber length the power of the beam is less than the critical power $P_{\mathrm{c}}$. A maximum stable pulse compression is therefore expected for $P=P_{\mathrm{c}}$ : $\tau_{0} / \tau \approx 1+3.6 \pi d^{2} / g \zeta$

When the peak power or the fiber length increases, stable compression of the pulses increases until SRS occurs. The maximum compression factor is almost independent of the fiber length, only the factor 16 of eq. (11) is slightly length dependent. For practical purposes this means that the maximum compression with stable pulses is approximately 16 .

\section{Experimental setup}

The setup of our system is given in fig. 1. The mode-locked Nd:YLF laser produces 50 ps pulses at a repetition rate of $81.25 \mathrm{MHz}$. It is of utmost importance that our system has high amplitude and phase stability. Therefore two isolation stages are used to prevent any back reflection into the laser which causes instabilities. The first isolation stage, consisting of a cube polarizer and a Faraday rotator, is also used as a variable attenuator, using the half wave plate and the cube polarizer to change the input intensity on the fiber. The second isolator consists of a cube polarizer and a quarter wave plate. The quarter wave plate behind the fiber is used to match the polarization to maximum grating reflectivity.

The light is coupled into the fiber with a $10 \times \mathrm{mi}-$ croscope objective and recollimated again with a $10 \times$ objective at the end of the fiber. The overall trans-

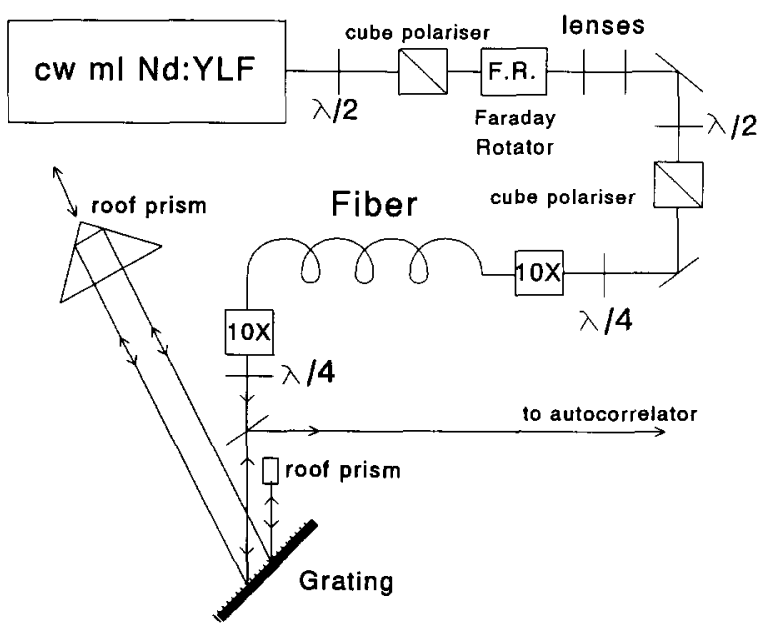

Fig. 1. Experimental setup of the optical pulse compressor. 
mission of the fiber is $55 \%$, mainly determined by incoupling losses.

The dispersive element is a grating which is used in a four-pass configuration as is indicated in fig. 1 to obtain a negative dispersive path. The distance between the grating and the prism determines the amount of negative GVD, and therefore also the pulse length. This distance is easily tunable. The upper limit of the distance between the roof prism and the grating is about $1 \mathrm{~m}$. The grating used is an 1800 lines/ $\mathrm{mm}$ holographic grating with $90 \%$ reflection in the minus first order at $75^{\circ}$ angle of incidence.

A background-free autocorrelation measurement technique using a $\mathrm{LiIO}_{3}$ crystal is used to measure the pulse width. For the deconvolution of this second harmonic generated (SHG) signal a sech ${ }^{2}$ pulse shape is assumed.

The experiments are carried out for various fiber lengths varying from $5 \mathrm{~m}$ to $125 \mathrm{~m}$. For each fiber length the compressed pulse width is measured as a function of transmitted power through the fiber. For each measurement the distance between the roof prism and the grating is optimized to achieve haximum compression.

\section{Results}

In fig. 2 the measured compression factor as a function of output power for various fiber lengths is shown. For compression factors lower than about seven the measured pulse width is larger than expected. These compression factors were limited by the maximum distance of $1 \mathrm{~m}$ between the roof prism and the grating (see also fig. 5). The fact that this occurred for the same compression factor for all different fibers also shows that laser power and fiber length are indeed interchangeable. From this figure it is also clear that the compression saturates at powers where SRS becomes important. The compression factor where SRS starts up decreases for shorter fiber length. This shows the dependence of the factor 16 of eq. (11) on the fiber length, indicated in fig. 2. Depletion of the input beam has also been observed in the region where SRS shows up.

For all measurements the grating-prism distance is optimized. This is shown in fig. 3. The four-pass grating setup used is equal to two dispersive lines as

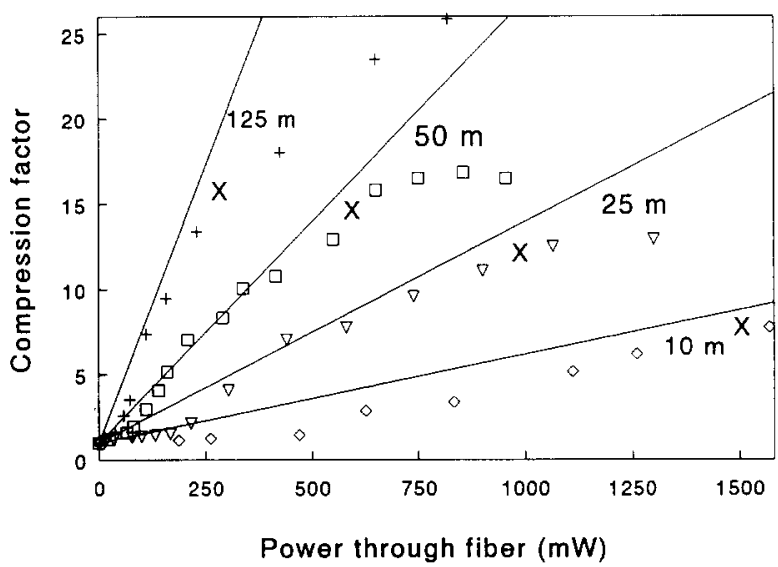

Fig. 2. Behaviour of the compression factor as a function the average power behind the fiber for various fiber lengths. The straight lines represent the theoretical predictions of eq. (2). The points indicated with $\mathrm{X}$ represent the power where SRS starts to influence the compression factor.

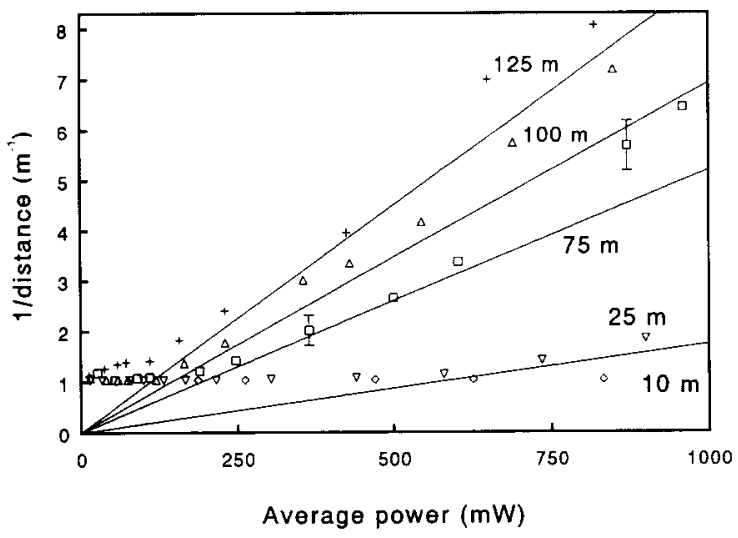

Fig. 3. The inversed grating-prism distance for optimized pulse compression is shown as a function of the average power after the fiber for various fiber lengths. The theoretical predictions are indicated by the straight lines.

described in the theory. In these dispersive lines is one grating replaced by a prism which reflects the beam onto the grating again. In this way the distance between the grating and the prism is half the gratinggrating distance. Due to these two effects the grating-grating distance $b$ from eq. (10) is four times the grating-prism distance. From fig. 3 it can be seen that relation $(10)$ is valid between the limit given by the available grating-prism distance which is about $1 \mathrm{~m}$ and the limit where SRS occurs which is about 
$25 \mathrm{~cm}$. Below $25 \mathrm{~cm}$ the grating-prism distance tends to decrease faster than when only SPM is present. The error bars in this figure are about $20 \%$ which shows that the compressor is not sensitive to the exact distance.

Because in all equations the output power and the fiber length are interchangeable, it is interesting to look at pulses having equal compression factors for different fiber lengths. This is shown in fig. 4. The $25 \mathrm{~m}$ and $50 \mathrm{~m}$ fiber lengths show the expected substructure. The substructure for a $100 \mathrm{~m}$ fiber is very small compared to that of the other fiber lengths which means that GVD is not negligible for fiber lengths longer than approximately $100 \mathrm{~m}$. The influence of the GVD is still very small and only influences the substructure and not the pulse width.

Furthermore, it can be seen from fig. 4 that the shape of the large wings is independent of the fiber length. These wings can be reduced using either a nonlinear birefringence background reduction method $[8,9]$ or by spectral windowing after the reflection on the grating [10]. Both methods eliminate the spectral components at the edges of the special distribution which create the wings.

Figure 5 shows the pulse shape for different grating-prism distances for a certain laser power and fiber length. For distances smaller than the optimum distance a broadening of the pulse is seen while for larger distances the pulse shows more unwanted substructure. This gives an easy way to tune the pulse

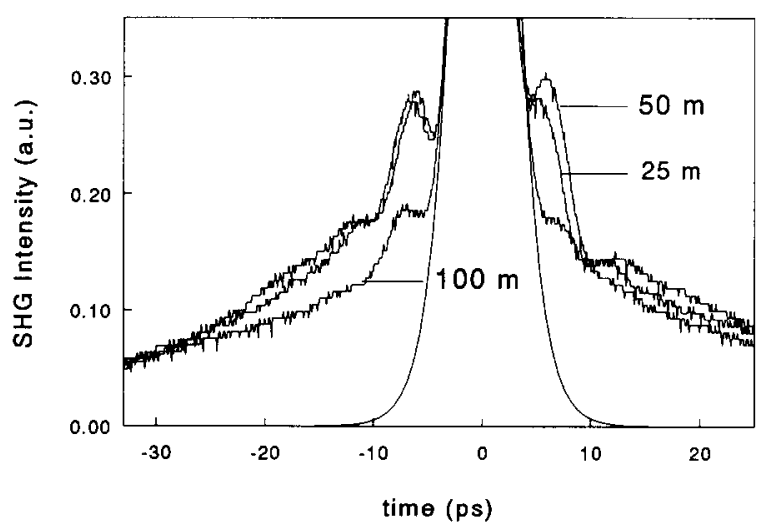

Fig. 4. In the case of equal compression factors ( $\sim 10$ times) the substructure of the puise is shown for fiber lengths of 25,50 and $100 \mathrm{~m}$. The theoretical $\operatorname{sech}^{2}$ function is also shown for comparison.

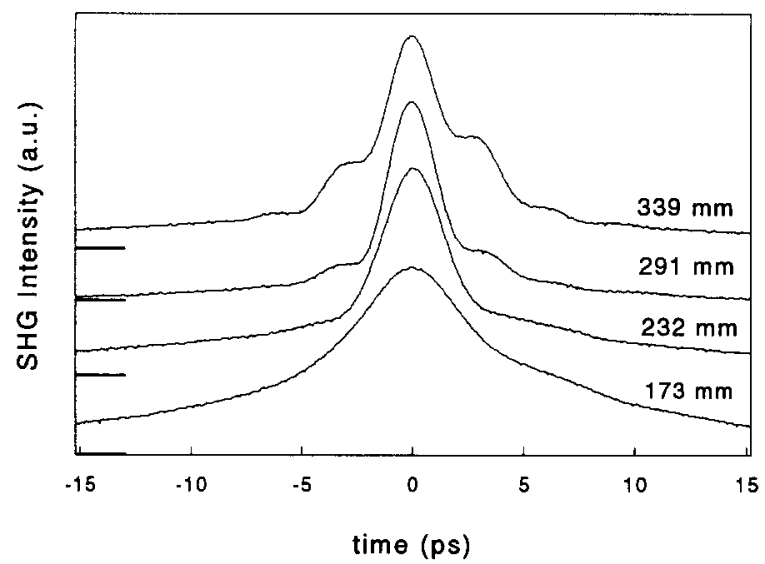

Fig. 5. For a single power ( $565 \mathrm{~mW}$ ) and fiber length ( $75 \mathrm{~m}$ ) the grating-prism distance is changed. This distance is indicated in the figure. The zero offsets for the different curves are indicated at the left axis.

length: just decrease the grating-prism distance from the optimum distance. In this way it is possible to tune the pulse length from 2 ps to 50 ps. Pulses obtained in this way remain their spectral width and accommodate a frequency chirp.

\section{Conclusion}

In summary, we systematically investigated the properties of a fiber-grating setup as a possibility for a pulse length tunable device. The theory for zero group velocity dispersion is valid for the available grating-prism distances. We found stimulated Raman scattering, starting at a critical power to be the limiting factor for stable pulse compression. A maximum compression factor of about 25 times is measured.

For long fiber lengths group velocity dispersion is present but the theory for zero group velocity dispersion still can be applied to a good approximation. The advantage of the presence of group velocity dispersion for long fiber lengths is the improved beam quality.

Finally, the pulse length can easily be tuned by changing the grating-prism distance without changing other parameters. 


\section{References}

[1] G.J. Ernst, W.J. Witteman, J.W.J. Verschuur, E.H. Haselhoff, R.F.X.A.M. Mols, A.F.M. Bouman, J.I.M. Botman, H.L. Hagedoorn, J.L. Delhez and W.J.G.M. Kleeven, Nucl. Instr. and Meth. A (1992) accepted for publication.

[2] J.S. Fraser and R.L. Sheffield, IEEE J. Quantum Electron. QE-23 (1987) 1489.

[3] D. Strickland and G. Mourou, Optics Comm. 56 (1985) 219.

[4] R.L. Fork, C.H. Brico Cruz, P.C. Becker and C.V. Shank, Optics Lett. 12 (1987) 483.
[5] A.E. Siegman, Lasers (University Science Books, Mill Valley, 1986) p. 387.

[6] W.J. Tomlinson, R.H. Stolen and C.V. Shank, J. Opt. Soc. Am. B 1 (1984) 139.

[7] R.G. Smith, Appl. Optics 11 (1972) 2489.

[8] D.C. Nguyen, D.E. Watkins and M.E. Weber, SPIE 895 (1988) 110.

[9] H.J. Halas and D. Grischkowsky, Appl. Phys. Lett. 48 (1986) 823.

[10] R.N. Thurston, J.P. Heritage, A.M. Weiner and W.J. Tomlinson, IEEE J. Quantum Electron. QE-22 (1986) 682 